\title{
The challenges of quantitative evaluation of a multi-setting, multi-strategy community-based childhood obesity prevention programme: lessons learnt from the eat well be active Community Programs in South Australia
}

\author{
Annabelle M Wilson ${ }^{1, *}$, Anthea M Magarey ${ }^{1}$, James Dollman ${ }^{2}$, Michelle Jones ${ }^{3}$ and \\ Nadia Mastersson ${ }^{3}$ \\ 'Department of Nutrition and Dietetics, Flinders University of South Australia, GPO Box 2100, Adelaide 5001, \\ South Australia: ${ }^{2}$ School of Health Sciences, University of South Australia, City East Campus, Adelaide, South \\ Australia: ${ }^{3}$ eat well be active Community Programs, Southern Primary Health, Noarlunga Centre, South Australia
}

Submitted 30 November 2008: Accepted 12 August 2009: First published online 13 October 2009

\begin{abstract}
Objective: To describe the rationale, development and implementation of the quantitative component of evaluation of a multi-setting, multi-strategy, community-based childhood obesity prevention project (the eat well be active (ewba) Community Programs) and the challenges associated with this process and some potential solutions.

Design: ewba has a quasi-experimental design with intervention and comparison communities. Baseline data were collected in 2006 and post-intervention measures will be taken from a non-matched cohort in 2009. Schoolchildren aged 10-12 years were chosen as one litmus group for evaluation purposes.

Setting: Thirty-nine primary schools in two metropolitan and two rural communities in South Australia.

Subjects: A total of 1732 10-12-year-old school students completed a nutrition and/ or a physical activity questionnaire and 1637 had anthropometric measures taken; 983 parents, 286 teachers, thirty-six principals, twenty-six canteen and thirteen outof-school-hours care (OSHC) workers completed Program-specific questionnaires developed for each of these target groups.

Results: The overall child response rate for the study was $49 \%$. Sixty-five per cent, $43 \%, 90 \%, 90 \%$ and $68 \%$ of parent, teachers, principals, canteen and OSHC workers respectively, completed and returned questionnaires. A number of practical, logistical and methodological challenges were experienced when undertaking this data collection.

Conclusions: Learnings from the process of quantitative baseline data collection for the ewba Community Programs can provide insights for other researchers planning similar studies with similar methods, particularly those evaluating multi-strategy programmes across multiple settings.
\end{abstract}

Keywords
Community-based obesity prevention
Evaluation
Childhood

The prevalence of overweight and obesity in school-aged children is estimated to be $10 \%$ worldwide and increasing $^{(1)}$. Obesity is recognised as a rapidly growing threat to the health of populations in an increasing number of countries around the world ${ }^{(2)}$, placing significant burden on health-care systems. Prevention is recognised as the most realistic and cost-effective strategy to deal with childhood obesity ${ }^{(3)}$.

While positive energy balance leads to the accumulation of excess weight, the aetiology of obesity is complex and dependent on more than just biology. For example, increased energy intake and/or decreased energy expenditure is commonly entwined with environmental factors, across multiple settings ${ }^{(4)}$.

There has been a call for community-based obesity interventions as a strategy for prevention of childhood obesity $^{(2)}$. Community-based interventions recognise the depth of community understanding held by members and their knowledge of community resources and dynamics $^{(5)}$. These provide the foundation when designing and delivering interventions, including choice of settings and strategies. Furthermore, individual behaviours are only sustained if they are carried out in an environment that supports healthy choices ${ }^{(5)}$. In the case of obesity, this 


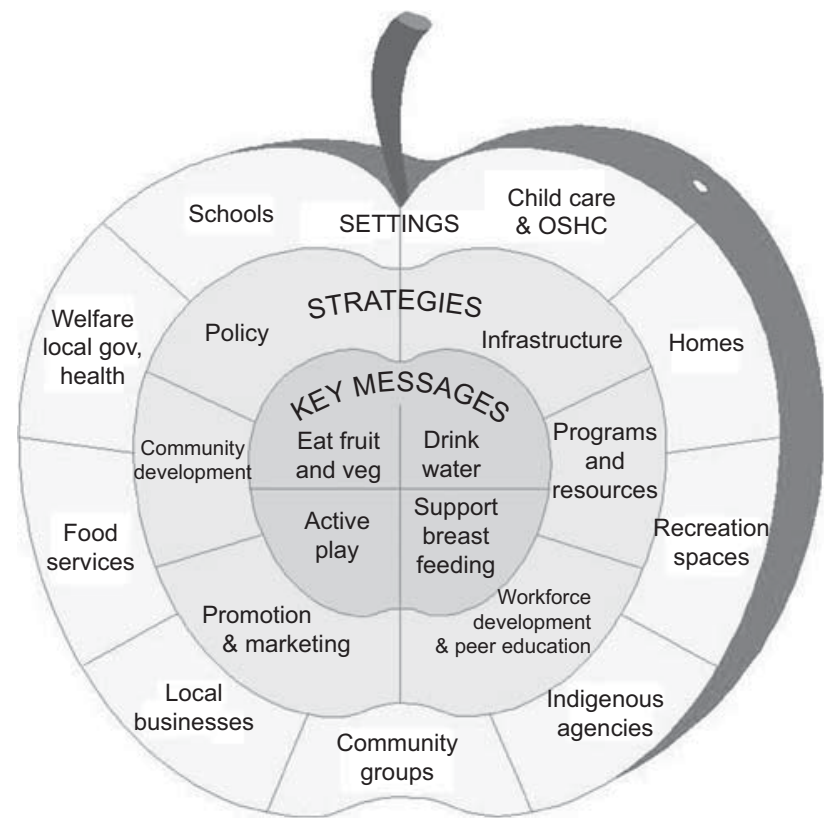

Fig. 1 Key messages, strategies and settings of the eat well be active Community Programs

means that individual behaviours associated with excess weight gain (including healthy eating and physical activity) must be addressed in the context of the environment and the societal and cultural factors relevant to the individual $^{(6)}$.

Despite the recognition that multi-setting, multi-strategy community-based action should be the foundation of obesity prevention efforts ${ }^{(7)}$, there are few published examples of such interventions ${ }^{(5)}$. The majority of childhood obesity prevention interventions are based in single settings, predominantly schools ${ }^{(8)}$. Therefore, there is a clear need to develop an evidence base of effective community-based obesity prevention interventions. Historically, insufficient priority has been placed on appropriate evaluation designs or insufficient funding provided to allow rigorous evaluation $^{(9)}$. Further evaluation of community-based interventions is hindered by the complexity of communities ${ }^{(9)}$, the inability to reproduce controlled environments often used in medical-based research and the lack of setting-specific tools appropriate to specific target groups that measure the outcome, process and impact of interventions.

The eat well be active (ewba) Community Programs in South Australia (SA) are community-based childhood obesity prevention interventions which address environmental and individual barriers to behaviour change through a portfolio of strategies in a range of settings (Fig. 1). The Programs are funded by SA Health (government) for 5 years, in response to the rising levels of childhood overweight and obesity and the need to obtain evidence of the effectiveness of community-based obesity prevention. The Programs aim to promote healthy weight in children and young people aged 0-18 years, and their families, through increasing healthy eating and physical activity behaviours. The ewba Community Programs have a rigorous evaluation framework that will contribute to the evidence regarding best practice for community-based childhood obesity prevention programmes. This framework includes quantitative and qualitative evaluation of process, impact and outcome elements and has a large scope across multiple population groups. The framework was designed to reflect the ewba interventions that were developed from a combination of best available evidence and extensive community consultation.

The present paper focuses on the rationale, development and implementation of the baseline data collection in schools, a major component of the quantitative evaluation of the ewba Community Programs. It identifies a number of challenges experienced during this process and suggests some solutions.

\section{Methods}

\section{Rationale}

Structure of the ewba Evaluation Academic Team

The ewba Evaluation Academic Team has two internal members; the ewba Evaluation Coordinator (responsible for coordinating and compiling data collection and evaluation processes) and the ewba Manager who oversees this process; and three external academics who advise the team in nutrition, physical activity, childhood obesity and community development. Additional research staff (e.g. a PhD Candidate) are also members of this team. The internal members of the Evaluation Academic Team work closely with the Project Coordinators who implement the project; however these Project Coordinators are not part of the Evaluation Team.

\section{Selection of intervention and comparison sites}

The intervention including the metropolitan suburb of Morphett Vale in southern Adelaide and the Rural City of Murray Bridge were selected by SA Health and the community health services implementing the projects, in consultation with community stakeholders, based on their high levels of disadvantage and the presence of existing infrastructure and experience necessary to support the Programs ${ }^{(10)}$. Two comparison communities (metropolitan suburbs of the Sea and Vines Department of Education and Children's Services (DECS) district and the Port Pirie Regional Council Area) were selected to match the sociodemographic profiles of the intervention sites as closely as possible, including: number and age distribution of children, socio-economic status as measured by the Index of Relative Social Disadvantage (IRSD), educational levels, occupational and income distributions, family sizes, ethnic mix and rural-urban mix ${ }^{(10)}$. The IRSD provides an indication of socio-economic disadvantage by ranking different geographic areas of Australia according to a 'score' that is created for the area based on 
characteristics of people, families and dwellings within that area $^{(11)}$.

\section{Selection of samples for evaluation}

Middle and upper primary-school children in school years five to seven (10-12-year-olds) were chosen as one litmus group for evaluation because: (i) there is a significant dose of ewba intervention delivered through the school setting; (ii) middle and upper primary-school children are cognitively able to complete simple written questionnaires and self-report dietary ${ }^{(12)}$ and physical activity habits ${ }^{(13)}$; and (iii) the primary-school curriculum is reasonably able to accommodate the time required for data collection. School students, their parents, school principals, teachers, canteen and out-of-school-hours care (OSHC) managers at intervention and comparison schools were invited to participate in the evaluation.

Selection of anthropometric outcomes for measurement Outcomes were chosen in consultation with the ewba Evaluation Team. Height and weight were measured and BMI was calculated. Waist circumference was taken as a surrogate measure of central abdominal adiposity based on the following rationale. First, waist circumference is an indirect measure of central adiposity, which is strongly correlated with risk for CVD in adults ${ }^{(14)}$ and an adverse lipid profile and hyperinsulinaemia in children ${ }^{(15)}$. Second, children's waist circumference correlates well with computerised tomography scan as a measure of subcutaneous abdominal adipose tissue $(r=0 \cdot 93)$, and fairly well with intra-abdominal adipose tissue $(r=0 \cdot 84)^{(16)}$.
Third, waist circumference is easy to measure with simple, low-cost equipment, has low observer error, offers good reliability, validity and low measurement error ${ }^{(17)}$ and has been used as a measure of child central adiposity in similar obesity prevention projects in Australia ${ }^{(18)}$.

\section{Development of tools for quantitative ewba evaluation}

The key messages and objectives of ewba (Fig. 1) informed the selection of the nutrition and physical activity outcomes to be measured. These outcomes included behaviours and also attitudes, knowledge and environments, which influence these behaviours. It was acknowledged that traditional methods of dietary and physical activity assessment (e.g. diet diaries and accelerometers) do not provide insight into attitudes, knowledge and environments but such information is invaluable to understand the obesity epidemic.

Due to the lack of tools in the published literature that addressed both the breadth of enquiry of ewba (Fig. 1) and the specific project goals, more contemporary evaluation questions and methods that encompassed these factors were specifically developed through consultation and review. Several unpublished questionnaires not yet tested for validity or reliability from similar interstate projects helped inform the content of these questionnaires. Selfreport questionnaires were chosen based on cost and time effectiveness, lower respondent burden and the age range of the sample (10-12 years) being appropriate for selfreporting of dietary and physical activity behaviours ${ }^{(12,13)}$.

The seven questionnaires (Table 1) measure the obesogenicity of one or more of the home, school and

Table 1 The seven Program-specific eat well be active questionnaires for evaluation purposes in intervention and comparison sites

\begin{tabular}{|c|c|c|c|}
\hline Questionnaire & Completed by whom & Number of items & Key measures \\
\hline Nutrition & $\begin{array}{l}\text { Students in school years } \\
5-7 \text { (age 10-12 years) }\end{array}$ & 12 & $\begin{array}{l}\text { - Child behaviours, attitudes and knowledge associated } \\
\text { with HE } \\
\text { - Obesogenicity of home, school and community } \\
\text { environments* }\end{array}$ \\
\hline PA & $\begin{array}{l}\text { Students in school years } \\
5-7 \text { (age 10-12 years) }\end{array}$ & 16 & $\begin{array}{l}\text { - Child behaviours, attitudes and knowledge associated } \\
\text { with PA } \\
\text { - Obesogenicity of home, school and community } \\
\text { environments* }\end{array}$ \\
\hline Parent & $\begin{array}{l}\text { Parents of students in } \\
\text { school years } 5-7\end{array}$ & 27 & $\begin{array}{l}\text { - Demographics } \\
\text { - Obesogenicity of home environments* } \\
\text { - Parental knowledge and attitudes towards HE and PA } \\
\text { - Child PA and HE behaviours }\end{array}$ \\
\hline Teacher & Primary-school teachers & 15 & $\begin{array}{l}\text { - Teaching practices around HE and PA and inclusion in } \\
\text { school curriculum } \\
\text { - Training/experience in HE and PA } \\
\text { - Teacher knowledge and attitudes towards HE and PA }\end{array}$ \\
\hline Principal & Principals & 27 & $\begin{array}{l}\text { - School HE and PA environments* } \\
\text { - Links with parents/other organisations around HE and PA }\end{array}$ \\
\hline Canteent & Canteen manager & 16 & $\begin{array}{l}\text { - Canteen operational details } \\
\text { - Factors affecting food sold } \\
\text { - Food sold by canteen, including healthier products }\end{array}$ \\
\hline OSHC+ & OSHC manager & 20 & $\begin{array}{l}\text { - OSHC HE and PA environments* } \\
\text { OSHC manager knowledge and attitudes towards HE } \\
\text { and PA }\end{array}$ \\
\hline
\end{tabular}

$\mathrm{HE}$, healthy eating; PA, physical activity; OSHC, out-of-school-hours care. ${ }^{*}$ Physical, political, socio-cultural and financial environments ${ }^{(18)}$. tNot all schools have these facilities. 
community environments. This enables triangulation of data to test for agreement between reports (e.g. student and parent report). Four types of environments were investigated in all settings - political, physical, financial and sociocultural ${ }^{(19)}$.

All seven questionnaires were piloted with small convenience samples of the relevant groups, in areas external to the ewba sites, prior to their use. The child nutrition questionnaire has been shown to be valid and reliable ${ }^{(20)}$ and can be accessed from http://www.ijbnpa.org/content/5/1/5. The child physical activity, teacher and parent questionnaires are currently being assessed for validity and reliability.

\section{Implementation}

Selecting and contacting schools

The process and timelines for contacting schools regarding participation in the ewba intervention and collection of baseline data are outlined in Fig. 2.
All government, catholic and independent primary schools in the intervention sites were invited to participate in the ewba intervention and evaluation. Those in comparison sites were invited to participate in the evaluation only. Schools in the intervention sites were offered the portfolio of ewba strategies that promoted healthy eating and physical activity and individually chose which strategies they each implemented throughout the intervention period. As a benefit of participation in data collection, both intervention and comparison schools were provided with aggregate information from all participating schools on students' nutrition, physical activity and standardised BMI, and summarised policy and practice patterns.

\section{Staff training}

A team of fourteen staff were recruited and trained to collect the baseline data (Fig. 2). All staff attended a $1 \mathrm{~d}$ training session run by the ewba Evaluation Academic

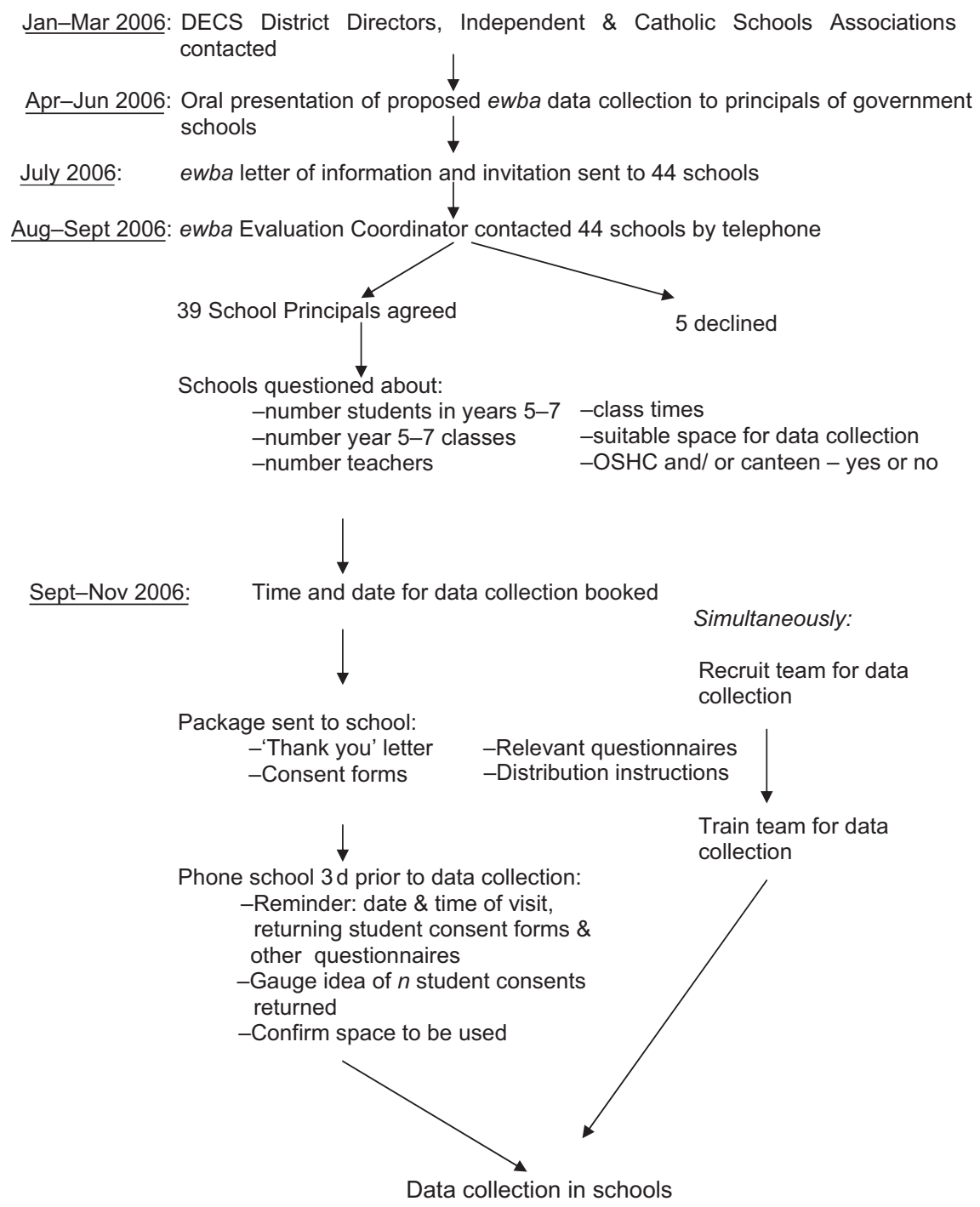

Fig. 2 Process used for contacting schools about the ewba intervention and baseline data collection 
Team. This included training in body image sensitivity ${ }^{(21)}$, measurement of weight, height and waist circumference, and description and practical run-through of the student questionnaires.

Staff were also trained to use three other resources including a standard preamble to introduce the data collection process and to provide instruction on completion of the student questionnaires, a poster depicting serve sizes of fruit and vegetables to assist students with portion size estimation in the nutrition questionnaire and a series of visual aids to assist students distinguish between organised and nonorganised activities in the physical activity questionnaire.

\section{Obtaining parental consent and child assent}

All year five, six and seven students in participating schools were invited to participate in data collection and consent forms were sent to participating schools to distribute to parents (Fig. 2). Sample size calculations indicated that a response rate of $60 \%$ would enable a $20 \%$ change in prevalence of a range of nutrition and physical activity behaviours to be detected with $80 \%$ power and alpha 0.05 .
Students returned consent forms with parental consent and child assent to their teachers and these were collected on the day of data collection. Students could consent to and participate in questionnaires but not anthropometric measures and vice versa, and they could withdraw from participation at any time. Verbal parental consent was accepted over the telephone on the day of measurement, provided the completed consent form was returned to ewba at a later date. Due to a low return rate of consent forms in the first eleven schools measured in School Term 3, all students returning a consent form (regardless of their consent to the measures) in the subsequent twenty-eight schools measured in School Term 4 were offered a small gift (a hacky sack), provided the school gave permission.

\section{Baseline data collection}

The process of baseline data collection is outlined in Fig. 3.

Questionnaires. Prior to distribution of the questionnaires, the Measurement Team Leader read out the standard preamble to the whole student group. To avoid any systematic bias resulting from good student
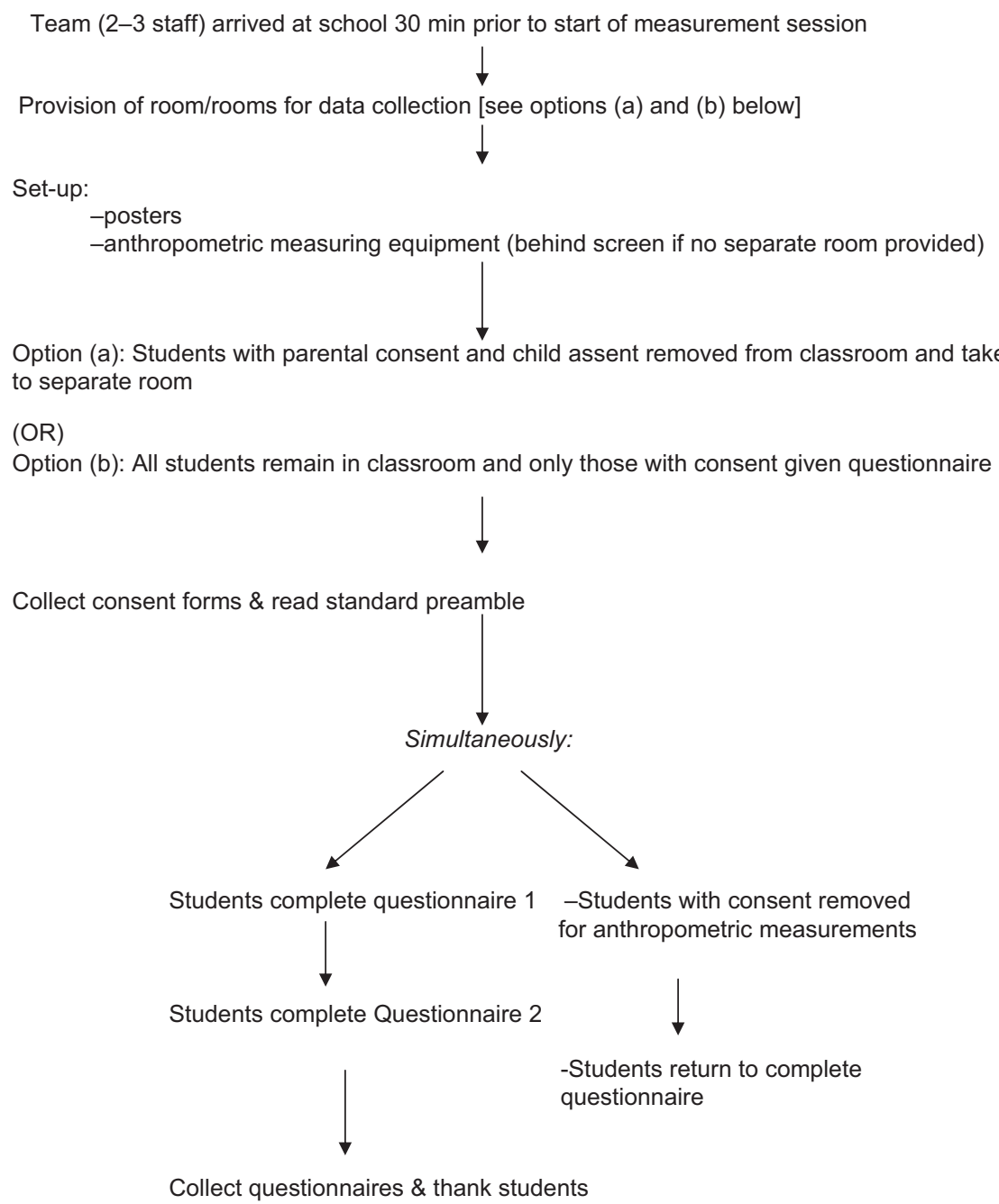

Fig. 3 Process of ewba baseline data collection in schools 
Table 2 Body Image Protocol used in the eat well be active Community Programs ${ }^{(20)}$

Elements of the ewba Body Image Protocol

- Parent consent and child assent required for child participation

- Information accompanying consent form explained the population approach of ewba and thus focus was on group not individual results - hence individual results not released

- Measurement staff trained in body image sensitivity by an external expert to ensure consistency of language and reduce transfer of negative messages about weight

- Police criminal record check for all measurement staff

- Measurements conducted out of view of other students and results screened from participants to reduce opportunities for comparison

- Children asked only to remove shoes and any heavy weight jumpers/jackets

- Waist measurements taken over the child's shirt

- Participants able to choose whether measurements taken by a male or female staff member

- School teacher to be present at all times during data collection and measurements

ewba $=$ eat well be active.

concentration during completion of the first survey and poorer concentration during completion of the second, the order in which the questionnaires were administered was varied between classes. Consequently, this effect (if present) was distributed across the two surveys. Students completed the questionnaires independently with the exception of two more complex questions in each questionnaire. These were led by the team leader with the whole student group, as piloting identified that students required extra assistance with these questions ${ }^{(20)}$. Staff referred to posters depicting fruit and vegetable serve sizes, and organised and nonorganised activities, when necessary.

Anthropometric measurements. The decision to take anthropometric measurements from children was carefully considered by the ewba Management Committee and Evaluation Team. In addition, informal consultation conducted with local stakeholder groups indicated majority support for the measurements to be taken whilst keeping in mind body image concerns.

A station for anthropometric measurements was set up with a set of scales (model HD319; Tanita, Tokyo, Japan), stadiometer (model PE087; Mentone Educational Centre, Melbourne, Australia) and tape measure for waist measurements (model W606PM; Lufkin, Albury, Australia). In the larger schools and when there was sufficient measurement staff, two stations were set up. If a separate room was not provided for anthropometric measures, the equipment was set up behind a screen in the same room as questionnaires were administered. In this case, students were out of view of other students when measured, but could still be in view of the observing teacher. One male and one female staff member were present to take measurements. Particular steps were taken to minimise body image concerns, based on the work of Gibbs et $a l^{(21)}$ (Table 2).

Students' names were called out and they were withdrawn temporarily from completing the questionnaires.
Height, weight and waist circumference were measured without shoes using the protocols recommended by the International Society for the Advancement of Kinanthropometry (ISAK) ${ }^{(22)}$. Waist circumference was measured at the level of the visible narrowing of the waist and at endtidal expiration ${ }^{(10)}$. All measurements were taken twice; a third was taken if the difference between the two measurements was too great (height: $>5 \mathrm{~mm}$; waist: if the difference exceeded $2 \%$ of the lower of the two scores; weight: if there was a $1 \%$ (or greater) difference between the first and second readings $)^{(22)}$. The mean of two and median of three measures were taken as the final score. All measurements were recorded on a standard record sheet. Inter-tester technical error of measurement (TEM) and intratester TEM were calculated for five of the measurers and shown to be well within acceptable ISAK standards ${ }^{(10,22)}$.

\section{Distribution of other questionnaires}

Parent. On the consent form for child measurements, parents were asked to indicate if they were happy to complete a parent survey and if so to provide their home address. Questionnaires were sent by ewba home to parents who returned them directly to ewba via replypaid envelopes. Parents who returned a completed questionnaire went into the draw to win one of twenty A $\$ 25$ shopping vouchers.

School. Teacher, principal, OSHC and canteen manager questionnaires were sent to the schools prior to data collection (Fig. 2). Ideally, the completed questionnaires were collected on the measurement day (Fig. 3) and if they had not been completed, extra copies were provided with a request to return to ewba as soon as possible. The five schools with the highest return rate of teacher and student questionnaires were offered an $\mathrm{A} \$ 100$ voucher for sports equipment, a water cooler or fruit and vegetables.

\section{Data entry and analysis}

As cost prohibited all data to be entered twice and checked for agreement (double data entry), a random sample of $10 \%$ of child nutrition, physical activity, teacher and parent questionnaires were checked by the ewba team. The scoring system used to assess validity and reliability of the child nutrition questionnaire ${ }^{(20)}$ was used to analyse the data from this questionnaire, and a similar scoring system was developed for the physical activity questionnaire. Target scores were developed for the nutrition ${ }^{(20)}$ and physical activity questionnaires and these were used to report meaningful frequency data at baseline. Frequency data on responses from parent, teacher, principal, canteen and OSHC questionnaires were also reported. SPSS version $12 \cdot 0 \cdot 1$ was used to analyse data.

\section{Ethics approvals}

Ethics approval was granted from the SA Health Human Research, the DECS and the South Australian Aboriginal Health Research Ethics Committees. 


\section{Results}

\section{Consent and response rates}

Of the forty-four primary schools in intervention and comparison sites, thirty-nine agreed to participate (89\% acceptance rate) ${ }^{(10)}$. The five schools that declined to participate were in comparison sites.

Table 3 shows response, consent and completion rates for the student questionnaires and anthropometry; and parent, teacher, principal, OSHC and canteen questionnaires. For students, consent rate is different from completion rate due to absences on the day of survey. The number of students returning the consent form where either the student or parent did not assent/consent to either questionnaires and/or measurement was 262. One hundred and ten students who had consented were absent on the day of survey. Twenty-three parents provided verbal consent on the day of survey. Table 4 shows the difference in overall response, consent and completion rates in the schools that received a gift for return of surveys (twenty-eight schools) compared with those who did not (eleven schools). Response rates were significantly higher in schools receiving the gift $(P<0 \cdot 001)$, and so were consent and completion rates $(P<0 \cdot 05)$. One school chose not to take up the option of the gift.

\section{Data entry}

Ten per cent of the child nutrition, child physical activity and teacher questionnaires were checked for data entry errors (173, 173 and twenty-nine questionnaires respectively). The error rates (expressed as number of items with an error per total number of items in one questionnaire) were found to be $0.3 \%, 0.5 \%$ and $0.17 \%$ respectively. Twelve per cent of parent questionnaires were checked (121 questionnaires). The error rate was found to be $0.69 \%$ with three questions regarding higher errors than any others. Exclusion of these three questions reduced the error value to $0 \cdot 48 \%$. These three questions were checked in all 983 questionnaires. The entire anthropometry data file was checked for errors and amended accordingly. Eleven students were excluded due to incomplete or missing data.

\section{Discussion}

The present paper describes the rationale, development and implementation of the quantitative baseline data collection in schools, which is one component of the evaluation of the ewba Community Programs. It focuses on the questionnaires and anthropometric measures taken in 10-12-year-old school students. This discussion will demonstrate the numerous logistical, practical and methodological challenges met during the data collection process, and will also consider solutions to these challenges, which provide learnings for other researchers involved in evaluation of similar programmes. To avoid biasing the results of the ewba evaluation, such adaptations will not be introduced in the present study at postintervention data collection.

There were large variations between schools in terms of daily timetable, class structure and school dynamics, and it was necessary to ensure that data collection slotted into the schools' schedules as seamlessly as possible. Similar projects should obtain school schedules as early as possible and ensure that their data collection process is flexible enough to fit in with differing school schedules.

Similarly, to ensure maximum return of consent rates and hence sample size, consent forms were not collected by ewba until the day of survey. Hence decisions that

Table 4 Completion, consent and response rates to surveys and anthropometric measures by students receiving a small gift for return of consent form compared with those not receiving a gift

\begin{tabular}{lcc}
\hline & $\begin{array}{l}\text { Schools with students } \\
\text { receiving gift (\%) }\end{array}$ & $\begin{array}{l}\text { Schools with students } \\
\text { not receiving gift (\%) }\end{array}$ \\
\hline Completion rate & $48 \cdot 6^{*}$ & $43 \cdot 1$ \\
Consent rate & $51 \cdot 6^{*}$ & $46 \cdot 3$ \\
Response rate & $60 \cdot 9^{* *}$ & $51 \cdot 5$ \\
\hline
\end{tabular}

${ }^{\star} P<0.05 ;{ }^{* *} P<0.001$

Table 3 Rates of return for the student, principal, out-of-school-hours care, canteen, parent and teacher questionnaires and student participation in the anthropometric measurements for baseline data collection of the eat well be active Community Programs

\begin{tabular}{|c|c|c|c|c|c|c|c|}
\hline \multirow[b]{2}{*}{ Questionnaire/measurements } & \multirow[b]{2}{*}{ Number eligible } & \multicolumn{2}{|c|}{ Responset } & \multicolumn{2}{|c|}{ Consent‡ } & \multicolumn{2}{|c|}{ Completed§ } \\
\hline & & $n$ & $\%$ & $n$ & $\%$ & $n$ & $\%$ \\
\hline Student nutrition & 3647 & 2104 & $57 \cdot 7$ & 1842 & $50 \cdot 5$ & 1732 & $47 \cdot 5$ \\
\hline Student PA & 3647 & 2104 & $57 \cdot 7$ & 1842 & $50 \cdot 5$ & 1732 & $47 \cdot 5$ \\
\hline Student anthropometric measurements & 3647 & 2009 & $55 \cdot 0$ & 1747 & $47 \cdot 9$ & 1626 & $44 \cdot 8$ \\
\hline Parent & 1519 & \multicolumn{2}{|c|}{ * } & \multicolumn{2}{|c|}{ * } & 983 & $65 \cdot 0$ \\
\hline Teacher & 667 & \multicolumn{2}{|c|}{ * } & \multicolumn{2}{|c|}{ * } & 286 & $43 \cdot 0$ \\
\hline Principal & 40 & \multicolumn{2}{|c|}{ * } & \multicolumn{2}{|c|}{ * } & 36 & $90 \cdot 0$ \\
\hline OSHC & 19 & \multirow{2}{*}{\multicolumn{2}{|c|}{$\begin{array}{l}* \\
*\end{array}$}} & \multicolumn{2}{|c|}{ * } & 13 & $68 \cdot 0$ \\
\hline Canteen & 29 & & & & & 26 & $90 \cdot 0$ \\
\hline
\end{tabular}

PA, physical activity; OSHC, out-of-school-hours care.

*Not applicable: completion of questionnaire taken as consent - no extra consent form required.

tReturned a consent form, regardless of whether consented to questionnaires and/or anthropometric measures (could return a form and not consent).

†Returned a consent form and consented to questionnaires and/or anthropometric measures.

$\S$ Questionnaires were completed and/or anthropometric measures were taken on the day of survey. 
were based on the number of children consenting could not be made until the day of survey. For example, if consents were low, it was less disruptive to combine students from multiple classes with consent in a separate space to complete questionnaires, while when consents were high, it was more practical to visit each class separately and students without consent were kept busy with another task. It is important that researchers in similar studies allow enough time and flexibility in their schedule to allow such options.

Data collection was performed late in School Term 3 and early in Term 4 (September to November) 2006. Term 4 is recognised as a busy time for schools and this could have had an impact on their response to data collection. If possible, researchers should avoid data collection in Term 4, particularly towards the end. However, the short period over which data were collected was ideal as it limited any seasonal changes in nutrition and physical activity behaviours that were independent of the programme.

The ability of teachers to control student behaviour varied and in some cases ewba staff were required to assist with behaviour management, making it more difficult to complete other tasks. Some students had very low literacy levels and often required a staff member to guide them through the questionnaires. This was resource intensive and left fewer staff to assist the remainder of the class with queries. In the early stages of data collection, it became apparent that students in year five generally required more assistance to complete questionnaires than those in years six or seven. Consequently, larger teams (four to five people) were allocated to attend classes with year five students. It is important that researchers in similar studies assess whether behavior management and literacy problems are likely to be an issue and if so, provide sufficient staff members to handle such situations effectively.

Designation of a suitable space for data collection by the school often proved problematic. In particular, anthropometric measures had to be taken in an area that was not secluded, but private enough for children to feel comfortable and not vulnerable. It is important that schools receive clear guidelines about the types of spaces that are and are not appropriate for such measurements. In case a suitable space is not available, the research team needs to be prepared with a backup, such as a screen that can be used as a privacy shield.

It is important to allow sufficient time between recruitment of measurement staff and schools and the commencement of measures (Fig. 2). In terms of measurement staff, sufficient time ensures detailed training around the questionnaires, in particular how to respond to specific student queries, ensuring consistent responses across team members. However, an in-depth understanding of the types of questions asked by students only develops over time by undertaking the data collection process. This highlights the importance of extensively piloting questionnaires where possible. In the case of time between recruitment of schools and measures, in some cases, there was only 6-8 weeks between the first letter schools received from ewba and data collection (Fig. 2). This may explain why the target completion rate of $60 \%$ was not reached. If possible, similar studies should allow more time between first contacting schools and data collection. This would allow greater flexibility with dates and times, provide opportunity for project staff to visit schools and identify suitable spaces for data collection, allow reminders/extra consent forms to be sent home and more time to collect information from schools including number of teachers, students and classes. Consent forms could also be collected prior to data collection, with additional consenters allowed on the day. However, such methods would require extra visits to the school, which would be more resource- and time-intensive.

The consent rates of students in the present study were similar to those in other studies. For example, $46 \%$ of $10-$ year-olds in intervention and control schools consented to baseline measures in the Energize project ${ }^{(23)}$. Fortyfour per cent of primary-school children in control sites and $58 \%$ in intervention sites consented to measures in the Be Active Eat Well intervention ${ }^{(18)}$.

The body image protocol, standard preamble and use of the small gift for students returning consent forms, all worked well during the baseline quantitative measures for ewba. These may also be beneficial strategies for similar studies. No specific issues around body image were raised, suggesting that the body image protocol was successful in this regard. The standard preamble was an effective introduction to the measuring team and questionnaires; no questions were consistently asked by students that indicated a lack of understanding of a certain question or instruction that should have been covered in the standard preamble. The use of the small gift for students returning consent forms (regardless of their consent to the measures) resulted in significantly higher response, consent and completion rates.

\section{Conclusion}

The present paper describes the rationale, development and implementation of one of the baseline quantitative data collection process as part of evaluation of a multi-setting, multi-strategy, community-based childhood obesity prevention programme. It demonstrates the complexity of developing a quantitative evaluation process in schools for a multi-strategy and multi-setting project, discusses some of the challenges associated with the data collection process and poses some solutions that may be considered by other similar projects. The experience of baseline data collection for the ewba Community Programs has provided a clearer understanding of the processes and potential difficulties involved with planning and implementing this type of evaluation. 
Similar programmes can use: (i) the quantitative evaluation of the ewba Community Programs as an example of one part of a rigorous evaluation for a community-based intervention to inform their own quantitative data collection; and (ii) the challenges and potential solutions reported in the present paper as a form of practical advice to assist with planning and implementing quantitative evaluation of similar, multi-setting, multi-strategy programmes.

\section{Acknowledgements}

The eat well be active Community Programs are funded by the Government of South Australia, SA Health and implemented by Southern Primary Health, of Southern Adelaide Health Service, and Murray Mallee Community Health, of Country Health SA. The authors declare that they have no conflict of interest. A.M.M., J.D. and N.M., in collaboration with Fiona Verity, developed the evaluation framework and project tools. A.M.W. and N.M. assisted in data collection. M.J. collated sociodemographic and consent data and wrote the ewba Baseline Evaluation Report, in conjunction with A.M.M., J.D., N.M. and Emma Clover, which was used when writing the present paper. A.M.W. wrote the first draft of the manuscript and it was revised by all authors. The authors acknowledge Terri Lamoree, ewba Evaluation Coordinator at the time of baseline data collection, who provided input into the present paper; in particular comments that informed the writing of the discussion. The authors also acknowledge the team of staff who collected baseline measurements and administered surveys, the schools, students and parents participating in the ewba Community Programs, Lily Chan and Emma Clover from the Flinders University Department of Nutrition and Dietetics for data checking and data analysis, Jerry Moller for input into the initial stages of the evaluation plan and Associate Professor Fiona Verity from the School of Social Work at Flinders University for her role on the ewba Evaluation Academic Team.

\section{References}

1. Lobstein T, Baur L \& Uauy R (2004) Obesity in children and young people: a crisis in public health. Obes Rev 5, Suppl. $1,4-85$.

2. World Health Organization (2004) Obesity: Preventing and Managing the Global Epidemic: Report of a WHO Consultation. Technical Report Series no. 894. Geneva: WHO.

3. Lobstein T, Baur L \& Uauy R (editors) (2003) Obesity in Young People - The Coming Crisis in Public Health. Report to the World Health Organization. London: WHO.

4. Dietz W \& Gortmaker S (2001) Preventing obesity in children and adolescents. Annu Rev Public Health 22, 337-353.

5. Economos C \& Irish-Hauser S (2007) Community interventions: a brief overview and their application to the obesity epidemic. J Law Med Ethics 35, 131-137.
6. Caballero BB (2004) Obesity prevention in children: opportunities and challenges. Int J Obes 28, Suppl. 3, S90-S95.

7. Bell C (2005) Community-based approaches to prevent obesity. In Food, Diet and Obesity, 1st ed., pp. 448-468 [D Mela, editor]. Washington, DC: Woodhead Publishing.

8. Summerbell CD, Waters E, Edmunds LD et al. (2005) Interventions for preventing obesity in children. Cochrane Database Syst Rev issue 3, CD001871.

9. Swinburn B, Bell C, King L et al. (2007) Obesity prevention programs demand high-quality evaluations. Aust $N Z J$ Public Health 31, 305-307.

10. Jones M, Magarey A, Dollman J et al. (2008) eat well be active Community Programs Evaluation Report Part 1: Baseline Data Collection. Adelaide: SA Health.

11. Pink B (2008) Information Paper: An Introduction to SocioEconomic Indexes for Areas (SEIFA) 2006. ABS Catalogue No. 2039.0. Commonwealth of Australia: Australian Bureau of Statistics.

12. Baranowski T \& Domel SB (1994) A cognitive model of children's reporting of food intake. Am J Clin Nutr 59, Suppl. 1, 212S-217S.

13. Welk G, Corbin C \& Dale D (2000) Measurement issues in the assessment of physical activity in children. Res $Q$ Exerc Sport 71, Suppl. 2, S59-S73.

14. Ross R, Fortier L \& Hudson R (1996) Separate associations between visceral and subcutaneous adipose tissue distribution, insulin and glucose levels in obese women. Diabetes Care 19, 1404-1411.

15. Freedman SE, Serdula MK, Srinivasan SR et al. (1999) The relation of circumferences and skinfolds to levels of lipids and insulin: the Bogalusa Heart Study. Am J Clin Nutr 69 , 308-317.

16. Goran MI, Gower BA, Treuth M et al. (1998) Prediction of intra-abdominal and subcutaneous abdominal adipose tissue in healthy pre-pubertal children. Int $J$ Obes 22, 549-558.

17. Power C, Lake JK \& Cole TJ (1997) Measurement and longterm health risks of child and adolescent fatness. Int J Obes 21, 507-526.

18. Sanigorski AM, Bell AC, Kremer PJ et al. (2008) Reducing unhealthy weight gain in children through communitycapacity building: results of a quasi-experimental intervention program, Be Active Eat Well. Int J Obes 32, 1060-1067.

19. Swinburn B, Egger G \& Raza F (1999) Dissecting obesogenic environments: the development and application of a framework for identifying and prioritizing environmental interventions for obesity. Prev Med 29, $563-570$

20. Wilson AM, Magarey AM \& Mastersson N (2008) Reliability and relative validity of a child nutrition questionnaire to simultaneously assess dietary patterns associated with positive energy balance and food behaviours, attitudes, knowledge and environments associated with healthy eating. Int J Behav Nutr Phys Act 5, 5.

21. Gibbs L, O'Connor T, Waters E et al. (2008) Addressing the potential adverse effects of school-based BMI assessments on children's wellbeing. Int J Pediatr Obes 3, 52-57.

22. Norton KI, Whittingham N, Carter JEL et al. (1996) Measurement techniques in anthropometry. In Anthropometrica, 1st ed. pp. 22-75 [K Norton and T Olds, editors]. Sydney: UNSW Press.

23. Graham D, Appleton S, Rush E et al. (2008) Increasing activity and improving nutrition through a schools-based programme: Project Energize. 1. Design, programme randomization and evaluation methodology. Public Health Nutr 11, 1076-1084. 